Virginia Commonwealth University

vCU Scholars Compass

2014

\title{
Nanoconfined water under electric field at constant chemical potential undergoes electrostriction
}

Davide Vanzo

Virginia Commonwealth University

D. Bratko

Virginia Commonwealth University, dbratko@vcu.edu

Alenka Luzar

Virginia Commonwealth University, aluzar@vcu.edu

Follow this and additional works at: http://scholarscompass.vcu.edu/chem_pubs

Cart of the Chemistry Commons

Vanzo, D., Bratko, D., \& Luzar, A. Nanoconfined water under electric field at constant chemical potential undergoes electrostriction. The Journal of Chemical Physics, 140, 074710 (2014). Copyright (C) 2014 AIP Publishing LLC.

\section{Downloaded from}

http://scholarscompass.vcu.edu/chem_pubs/64

This Article is brought to you for free and open access by the Dept. of Chemistry at VCU Scholars Compass. It has been accepted for inclusion in Chemistry Publications by an authorized administrator of VCU Scholars Compass. For more information, please contact libcompass@vcu.edu. 


\title{
Nanoconfined water under electric field at constant chemical potential undergoes electrostriction
}

\author{
Davide Vanzo, D. Bratko, ${ }^{\text {a) }}$ and Alenka Luzar ${ }^{\text {b) }}$ \\ Department of Chemistry, Virginia Commonwealth University, Richmond, Virginia 23284-2006, USA
}

(Received 26 October 2013; accepted 27 January 2014; published online 20 February 2014)

\begin{abstract}
Electric control of nanopore permeation by water and solutions enables gating in membrane ion channels and can be exploited for transient surface tuning of rugged substrates, to regulate capillary permeability in nanofluidics, and to facilitate energy absorption in porous hydrophobic media. Studies of capillary effects, enhanced by miniaturization, present experimental challenges in the nanoscale regime thus making molecular simulations an important complement to direct measurement. In a molecular dynamics (MD) simulation, exchange of water between the pores and environment requires modeling of coexisting confined and bulk phases, with confined water under the field maintaining equilibrium with the unperturbed environment. In the present article, we discuss viable methodologies for MD sampling in the above class of systems, subject to size-constraints and uncertainties of the barostat function under confinement and nonuniform-field effects. Smooth electric field variation is shown to avoid the inconsistencies of MD integration under abruptly varied field and related ambiguities of conventional barostatting in a strongly nonuniform interfacial system. When using a proper representation of the field at the border region of the confined water, we demonstrate a consistent increase in electrostriction as a function of the field strength inside the pore open to a field-free aqueous environment. () 2014 AIP Publishing LLC. [http://dx.doi.org/10.1063/1.4865126]
\end{abstract}

\section{INTRODUCTION}

Wetting propensity by water, and its phase behavior in nonpolar porous media, can be efficiently modulated by applying electric field. Field-induced nanopore permeation underlies the function of cell membrane channels ${ }^{1}$ and can provide a mechanism for flow control in nanofluidic devices. ${ }^{2}$ While it is possible to obtain first order estimates of pore wetting or dewetting from continuum descriptions, ${ }^{2,3}$ both static $^{4-7}$ and dynamic responses ${ }^{8-10}$ of nanoconfined water to electric field show significant quantitative or even qualitative differences from macroscopic predictions. Molecular simulations are well suited to explore these differences and underlying molecular mechanisms, which are often hard to approach by direct experiments. A number of recent simulation studies ${ }^{1,4-6,11,12}$ reached seemingly different conclusions about electric field effects on nanoconfined water, drawing attention to the essential role of imposed conditions. As discussed in Refs. 13 and 14, different conditions can lead to qualitatively different responses of bulk and confined water to the field. Specifically, the field enhances the density ${ }^{6}$ and stabilizes the liquid phase ${ }^{13,14}$ if the confinement is open to a field-free bath supplying extra molecules to preserve constant chemical potential. In other scenarios, the field can reduce the density $^{12,15}$ and increase the volatility ${ }^{12}$ of confined water. In addition to fixed state functions (e.g., amount of substance, volume, pressure, temperature, field strength, and chemical potential), it is essential to specify the region affected by the

\footnotetext{
a)dbratko@vcu.edu

b) aluzar@vcu.edu
}

field. Several practical problems, including electric control of nanofluidics, current regulation in nanopores, ${ }^{2}$ or energy absorption devices, ${ }^{16,17}$ involve aqueous confinements equilibrated with the surrounding bulk phase, such that both phases are characterized by equal chemical potentials. In this common scenario, the field is either limited to the confinement, or extended over the whole system including the bulk phase. In the former case, chemical potential is determined by the thermodynamic state of the environment, with any reduction in the chemical potential under the field being compensated by a density increase. If the field affects the environment as well, however, chemical potential typically decreases with increasing strength of the field. In the second scenario, the densities of both phases change with the field. The change, however, is different in each phase and the density can also be lowered compared to the field-free system. ${ }^{14,18}$

Clearly, electric control of nanowetting is most effective when the field is applied in the confinement without perturbing the surrounding reservoir. This setup is easily amenable $^{4,6,7,13}$ to Monte Carlo approaches, e.g., the Grand Canonical (GCMC) or Gibbs Ensemble (GMC) simulations, were nonphysical molecule exchanges enable modeling of the field-exposed confinement separately from the bulk phase. In Molecular Dynamics (MD), on the other hand, transfer of molecules between the two coexisting phases involves both regions in direct physical contact. Special care is warranted in modeling the interface between the unperturbed and fieldexposed domains. In the most direct approach, the field is introduced by internal charges, e.g., ionic species distributed on confining surfaces, or at ion channel orifices, to produce a field of desired strength and location. ${ }^{1,9,19-21}$ This technique 
captures realistic situations in ionized pores or ion channels, but is less appropriate for studies of adjustable fields between capacitor electrodes and becomes computationally demanding when the environment has to include an unperturbed bulklike region, making it necessary to extend the reservoir boundaries well beyond the range of charge-induced perturbations. The same concern applies to the more complex and compute intense constant-electrode-potential alternatives. ${ }^{22-24}$

A more efficient alternative is to impose a uniform applied field inside the confinement without concerning the underlying charge distribution (implicitly determined by the Poisson equation). This approach was used in an interesting study of field effects on confined water by Vaitheesavaran et al. ${ }^{5}$ however, their work did not explicitly address the conditions at the interface between the region affected by the field and the field-free environment. In the present article, we discuss the possible implementations of the approach and describe a viable treatment of the interface between the fieldexposed and unperturbed regions. As we show shortly, it is essential to treat the field at this interface self-consistently. We evaluate the artifacts associated with MD integration in a spatially discontinuous field. We then demonstrate a satisfactory MD implementation in model systems where we replace the stepwise change in the field strength by a smooth transition characterized by appropriately matched, gradually fading field components acting along, and normal to, the surface dividing the two uniform regions. We also discuss the use of conventional Nose-Hoower barostat ${ }^{25}$ in the presence of discontinuous field. We validate its performance through comparison with a system where the pressure is buffered by the inclusion of auxiliary vapor pockets coexisting with the liquid aqueous phase. ${ }^{26,27}$

Our model calculations show that water uptake in a confinement consistently increases with the strength of local electric field, conforming to the classical electrostriction behavior in agreement with previous Monte Carlo studies. ${ }^{6,7,13}$ The improvements we have made in modeling the interface between the field-exposed and unperturbed regions can explain the differences between our findings and the system behavior observed at equivalent conditions in an earlier MD approach. ${ }^{5}$

\section{MODELS AND METHODS}

\section{A. Model specifications}

We use a rectangular simulation box of size $L_{x} L_{y} L_{z}$ $\sim 62 \times 62 \times 50 \AA^{3}$, replicated periodically to mitigate finite size effects. For the sake of simplicity, we choose the origin of the coordinate system to coincide with the box center. Our model confinement, shown in Fig. 1(a), consists of a pair of circular platelets of $4 \mathrm{~nm}$ diameter at the separation $d=1.2 \mathrm{~nm}$, placed symmetrically above and below the plane $z=0$. The circular shape (Fig. 1(b)) minimizes the confinement/reservoir border area and removes any dependence of the confinement properties on the direction along the $(x, y)$ plane. The platelets are comprised of rigid hexagonal lattice mimicking reparameterized ${ }^{28}$ graphene, a standard wall material in computational studies of confined water., ${ }^{5,-31}$ The nanopore is surrounded by 4893 water molecules at

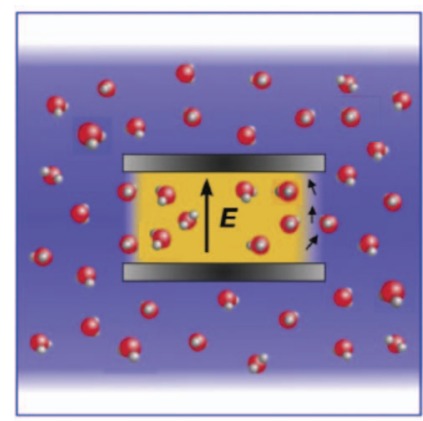

(a)

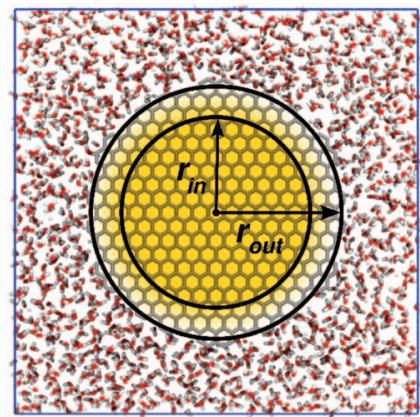

(b)
FIG. 1. (a) Cartoon of the simulated system showing the side view of the confinement consisting of a pair of disk-like platelets (grey) immersed in an aqueous reservoir. Blue color denotes unperturbed liquid water and yellow color corresponds to water under electric field; the arrows indicate the direction of the field. The system is periodically replicated in lateral directions $x, y$ (parallel to the disks) while it is closed by purely repulsive walls placed at the bottom and the top of the simulation box. White regions correspond to the pressure-buffering vapor pockets ${ }^{26,27}$ used in NVE simulations. Repulsive walls and vapor pockets were removed in simulations with Nose-Hoover barostating $(N P T)$, where the system was periodic in all three dimensions. (b) A top-view snapshot of the confinement showing the borders between the regions under homogeneous $\left(r<r_{\text {in }}\right)$, inhomogeneous $\left(r_{\text {in }} \leq r \leq r_{\text {out }}\right)$, and vanishing $\left(r>r_{\text {out }}\right)$ electric field.

vanishing external pressure, $P_{\mathrm{z}} \sim 0$. To enable consistent comparisons with previous works, ${ }^{1,5-7,19,28}$ we model water molecules using the extended simple point charge model (SPC/E). ${ }^{32-34}$ The model is well known for its satisfactory performance in studies of both interfacial and dielectric properties of water. ${ }^{35-39}$ The interaction between water oxygens and the atoms of the confinement platelets is described by the Lennard Jones $(\mathrm{LJ})$ potential. The LJ parameters of the platelet atoms, $\sigma_{\mathrm{CC}}=3.214 \AA$ and $\varepsilon_{\mathrm{CC}}=0.0231 \mathrm{kcal} \mathrm{mol}^{-1}$, correspond to System 17 from Table 3 of Werder et al. ${ }^{28}$ which we choose to mimic a strongly hydrophobic solid with contact angle $\theta_{\mathrm{c}} \sim 128^{\circ}$. At these conditions and no electric field, confined liquid state is metastable with respect to capillary evaporation. ${ }^{5,40-49}$ All nonelectrostatic interactions were truncated and shifted to zero at the cutoff distance $r_{\mathrm{c}}$ $=9 \AA$ and we use Lorentz-Berthelot mixing rules for crossinteractions. To evaluate any effect of water/water interactions across the graphene platelets, we also performed a few calculations using platelets of butylated graphane $\mathrm{s}^{50,51}$ (fully hydrogenated graphene), functionalized on the inner side of the plates. The distance of $\sim 15 \AA$, separating the 1 st hydration layers on the opposite sides of butylated graphane was more than twice bigger than in the case of graphene, effectively removing any correlations between these layers. Both materials were characterized in our previous work. ${ }^{50}$

To validate the barostat function in the presence of a steeply decaying field, we compare two different approaches. We use either the Nose-Hoover barostat ${ }^{25}$ embedded in our MD code, ${ }^{52}$ or the pressure-buffering method, ${ }^{26,27}$ which relies on the coexistence of liquid and vapor phases in the system. In the latter setup, two vapor pockets are created at the top and bottom of the simulation box situated between a pair of purely repulsive walls in analogy with previous works $^{26,27}$ (see Fig. 1(a)). In the system with the Nose-Hoover barostat, we apply the usual, three-dimensional periodic 
boundary conditions. The pressure-buffered system, on the other hand, is replicated only in lateral directions. We use the Yeh and Berkowitz adaptation ${ }^{53}$ of the Ewald sums to account for the lack of periodicity in the vertical $(z)$ direction. Repulsive walls in contact with the two vapor pockets interact with oxygen atoms of water through a harmonic potential with spring constant of $20 \mathrm{kcal} \mathrm{mol}^{-1} \AA^{-2}$. MD simulations are carried out by the LAMMPS simulation package in the $N P T$ ensemble at ambient pressure or, in the pressure-buffered case, in the $N V E$ ensemble. Because of vapor/liquid coexistence, the average pressure in the latter system corresponds to the saturated vapor pressure at given conditions. The temperature is maintained at $300 \mathrm{~K}$ by Nose-Hoover thermostat with 100 fs time constant. The NVE systems are run at essentially identical temperature, established by careful pre-equilibration at $N V T$ conditions. Velocity Verlet integrator is used with simulation time step 1 fs. Long-range electrostatic interactions are treated by particle-particle-particle mesh solver (PPPM) with a real-space cutoff of $9 \AA$ and relative precision tolerance in force per atom of $10^{-5}$.

\section{B. Applied electric field}

To model the behavior of a laterally homogeneous slab of field-exposed water, a uniform electric displacement field $\boldsymbol{D}(r, z)=\varepsilon_{o} \boldsymbol{E}^{0}(r, z)=\left(0,0, \varepsilon_{o} E_{z}^{0}(0,0)\right)$, perpendicular to the confining platelets is imposed inside the confinement. $\varepsilon_{o}$ is the permittivity of vacuum. The field could also be acquired by means of explicit wall charges, however, with the field due the charges propagating outside the confinement, the reservoir size should be increased to accommodate a domain of unperturbed bulk-like water. Generating a nearhomogeneous field with moderate finite-size effects in the confinement core would also require the use of bigger confining plates. Our approach avoids considerable computational costs associated with system-size increases, which would be required in the alternative scenario.

In view of the cylindrical symmetry of the confinement, we replace lateral coordinates $x$ and $y$ by the radial coordinate $r=\left(x^{2}+y^{2}\right)^{1 / 2}$. The vertical (z) component of the field, $E_{z}^{0}$, is constant in the confinement interior and vanishes in the surrounding bulk phase. The energy $U$ of interaction of water molecules with the applied electric field is given by the expression,

$$
U\left(\boldsymbol{X}_{i}\right)=\sum_{j} q_{j} \boldsymbol{r}_{j} \boldsymbol{E}^{0},
$$

where $\boldsymbol{X}_{\mathrm{i}}$ denotes the position and orientation of water molecule $i$ and index $j$ runs over the three partial charges $q_{j}$ of the molecule. At the interface between the confinement and the unperturbed reservoir, where the field is nonuniform, the appropriate relation is

$$
U\left(\boldsymbol{X}_{i}\right)=\sum_{j} q_{j} \psi\left(\boldsymbol{r}_{j}\right), \text { where } \boldsymbol{E}^{0}(\boldsymbol{r})=-\nabla \psi(\boldsymbol{r}) .
$$

$\psi(\boldsymbol{r})=\psi(r, z)$ is the contribution of the imposed field to the local electrostatic potential. Equation (2) also describes the ion-field interaction if pure water is replaced by salt solution. In our model system, a natural reference point for the imposed potential is the center of the box, i.e., $\psi(0,0)=0$, which by symmetry further implies that $\psi(\boldsymbol{r})$ vanishes for all lateral positions on the system midplane, $\psi(r, 0)=0$ for any $r$. Inside the region characterized by a homogeneous field $\boldsymbol{E}^{\mathrm{o}}$ with vanishing lateral components $E_{x}$ and $E_{y}$, electrostatic potential is determined solely by the height $z, \psi(r, z)=-z E_{z}^{0}(0,0)$. At the interface between the confinement and the field-free reservoir, however, the field depends on $r, \boldsymbol{E}^{\mathrm{o}}=\boldsymbol{E}^{\mathrm{O}}(r, z)$, and $\psi=\psi(r, z)$.

We consider two possible forms for the radial dependence of the imposed field at the interface between the two regions. In both cases we describe the transition from the homogeneous field in the confinement to vanishing field in the environment in terms of the decay function $f(r)$ :

$$
E_{z}^{0}(r, z)=E_{z}^{0}(0,0) f(r),
$$

with $f(r)$ equal to unity within the homogeneous region inside the confinement and zero in the bulk phase. The first of the two possible forms of the decay function $f(r)$ at the interface between the two regions describes the change in terms of a step function

$$
f(r)=1-\theta\left(r-r_{\text {out }}\right),
$$

where $\theta(x)$ is the Heaviside step function, which vanishes for negative $x$ and equals unity for positive $x$, hence the plane $r=r_{\text {out }}$ separates the domains with homogeneous fields $|\boldsymbol{E}|$ $=E_{z}^{0}$ and $|\boldsymbol{E}|=0$, in analogy with the system considered in Ref. 5. Alternatively, the field decay can be spread over a finite interval $r_{\text {in }} \leq r \leq r_{\text {out }}$ (Fig. 1(b)) of width of at least $O(d / 2)$, using a smooth function $f(r)=1$ for $r \leq r_{\text {in }}, f(r)=0$ if $r \geq r_{\text {out }}$, and $1 \geq f(r) \geq 0$ in between. Without any loss in generality, in the present study we employ the smooth decay function,

$$
\begin{array}{cl}
f\left(r_{s}\right)=\frac{1}{2}\left[\cos \left(\pi r_{s}\right)+1\right] & \text { if } r_{s} \leq 0 \\
0 & \text { if } 0 \leq r_{s} \leq 1, \quad r_{s}=\frac{r-r_{\text {in }}}{r_{\text {out }}-r_{\text {in }}} \\
\text { if } r_{s}>1 . &
\end{array}
$$

Fig. 2 illustrates the decay function from Eq. (5) and its derivative within the interval between $r_{\text {in }}$ and $r_{\text {out }}$. By avoiding the discontinuities in the electrostatic potential, this form simplifies a self-consistent integration of equations of motion in the transition region. The singularity introduced in Eq. (4) becomes apparent as we consider the radial component of the field, $E_{r}^{0}(r, z)=-\partial \psi(r, z) / \partial r$. While $E_{r}^{0}$ vanishes in homogeneous regions $\left(r<r_{\text {in }}\right.$ or $\left.r>r_{\text {out }}\right)$, the variation of $E_{z}^{0}$ at the interface implies the presence of nonzero radial field. Since $\boldsymbol{E}^{0}(r, z)=-\nabla \psi(r, z)$ represents a conservative vector field, the following relation has to be obeyed:

$$
\frac{\partial^{2} \psi(r, z)}{\partial z \partial r}=\frac{\partial^{2} \psi(r, z)}{\partial r \partial z} \text {, i.e., } \frac{\partial E_{r}^{0}(r, z)}{\partial z}=\frac{\partial E_{z}^{0}(r, z)}{\partial r} .
$$

For the selected form of the imposed field, Eq. (3),

$$
\frac{\partial E_{r}^{0}(r, z)}{\partial z}=E_{z}^{0}(0,0) \frac{\partial f(r)}{\partial r} .
$$

Since $E_{r}^{0}$ vanishes at $z=0$ and $E_{z}^{0}$ does not depend on $z$, integration of Eq. (6) gives

$$
E_{r}^{0}(r, z)=z E_{z}^{0}(0,0) \frac{\partial f(r)}{\partial r} .
$$



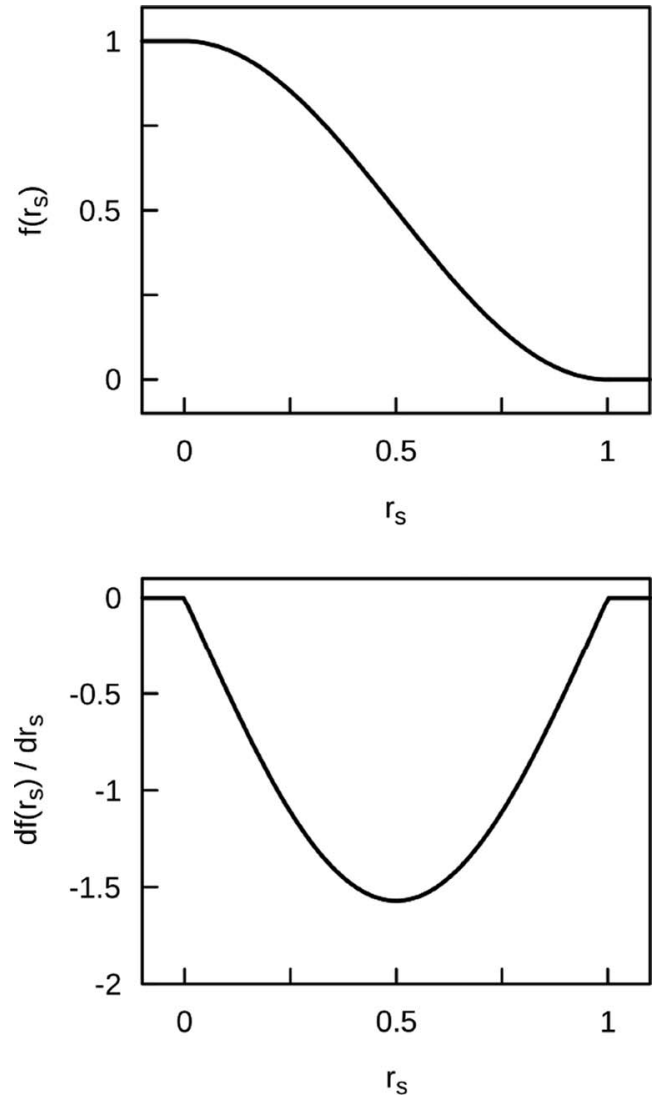

FIG. 2. Decay function $f\left(r_{s}\right)$ and its derivative within the interval $0 \leq r_{s}$ $\leq 1, r_{s}=\left(r-r_{\text {in }}\right) /\left(r_{\text {out }}-r_{\text {in }}\right)$. See Fig. 1(b).

For the special case described by Eq. (4)

$$
E_{r}^{0}(r, z)=-z E_{z}^{0}(0,0) \delta\left(r-r_{i n}\right)
$$

The magnitude of the radial component of the field at the interface between the two regions increases in proportion to the distance from the confinement midplane $z=0$. While the continuous form of $f(r)$ described by Eq. (5) allows straightforward integration of the equations of motion, the discontinuous field representation of Eq. (4) implies radial field of the form of the Dirac's impulse function $\delta\left(r-r_{i n}\right)$, which warrants the use of Discontinuous MD (DMD) ${ }^{54,55}$ In this technique, a molecule crossing the fielddiscontinuity plane acquires a force impulse changing its momentum and kinetic energy in proportion to the instantaneous change in the molecule's potential energy upon the crossing. The direction of the momentum change is given by the normal to the discontinuity plane. DMD is typically applied to systems with no continuous intermolecular forces. In these cases, linear molecular motion enables accurate predictions of future crossing events, ${ }^{54,55}$ allowing efficient trajectory calculation. To accommodate continuous intermolecular interactions and the discontinuous field simultaneously would require the combination of conventional MD and DMD, which is clearly impractical as curved molecular trajectories preclude analytic predictions of crossing events. Our trial calculations illustrate the consequences of ignoring the contribution of crossing events to the energy drift, the deviations in molecular partitioning between the reservoir and the con- finement, and pressure control in the inhomogeneous system. The use of smoothed field profile, on the other hand, offers a viable representation for MD studies and, as we will show shortly, supports the Nose-Hoover barostat while avoiding the need for discontinuity pressure-correction. ${ }^{25}$

\section{RESULTS AND DISCUSSION}

In this section, we compare the results of two different representations of the interface between the field-controlled confinement and field-free bath. We use systems with smooth and abrupt decays of the field at the confinement border to illustrate the consequences of the omission of the impulse term associated with the step-function representation.

To fix the chemical potential of water, the pressure in the reservoir is held close to zero by relying on two different techniques. In microcanonical $(N V E)$ simulations, performed to verify the accuracy of MD integration, the pressure is fixed at vapor pressure value due to the presence of two vapor pockets, placed at the top and bottom walls of the simulation box. ${ }^{26,27}$ Water structures from $N V E$ runs are compared with those obtained in parallel calculations in NPT ensemble with the NoseHoover algorithm ${ }^{25}$ used for temperature and pressure control. When using the latter method, confinement plates are held at fixed separation despite small fluctuations of the volume of the simulation box. In each of the two ensembles, we monitor the behavior of the system in the absence of external electric field and in two systems with field $\boldsymbol{E}=\left(0,0, E_{\mathrm{z}},\right)$ applied across the confinement. In one of the systems (system $S$ ), the field $E_{\mathrm{z}}$ decays smoothly over a finite width between radial distances $r_{\text {in }}=15 \AA$ and $r_{\text {out }}=19 \AA$ following Eq. (5). Trial calculations showed the smoothing interval can be varied without importantly affecting any of the properties of the confined fluid at $r<r_{\text {in }}$, or its equilibrium with the bulk phase. In the second system with nonzero field, (system $A$ ), the field $E_{\mathrm{Z}}$ is abruptly truncated at $r_{\text {out }}=19 \AA$ as described by Eq. (4). Using this model, we verify the performance of a standard MD algorithm without accounting for the impulse ${ }^{54}$ of electric force on charges crossing the field cutoff plane, Eq. (8). For random crossings and recrossings of molecular charges, the concomitant momentum changes should cancel out over a long observation time. However, this cancellation is no longer possible when molecules inside the confinement begin aligning with the field. For partially aligned molecules escaping from the confinement, the net effect of a crossing event is a momentum change, predominantly pointing in the inward direction. The magnitude of the change is determined from the condition of energy conservation, ${ }^{54,55}$ which requires a reduction in the molecule kinetic energy to compensate the loss of the favorable interaction with the field. If kinetic energy, associated with the radial velocity of the molecule, is not sufficient to overcome the loss of the favorable electrostatic energy, the crossing does not occur and the molecule bounces from the plane $r=r_{\text {out }}$. The radial field described in Eq. (8) is therefore expected to impede molecular escapes from the confinement, or facilitate the molecules' return after a successful escape. Approximate treatments, where the radial impulse field is neglected, ${ }^{5}$ can therefore underestimate the accumulation of dipolar molecules in the field-exposed region. We estimate 
the magnitude of this effect by comparing the systems with continuous and abrupt field truncation separately in $N V E$ and $N P T$ ensembles. But first we use the NVE simulation to test the consequences of the omission of the force impulse term on system's energy conservation.

\section{A. Energy conservation}

In this section we present the results for isolated systems' energies as a function of time during MD simulations in the microcanonical $(N V E)$ ensemble. In Fig. 3 we compare systems with no field (blue curve) and systems with smooth (system $S$, green) or abrupt field truncation (system $A$, red) during 2 ns runs, started after thorough equilibration in NVT ensemble at $300 \mathrm{~K}$.

The strength of the applied field $E_{z}^{0}(0,0)$ corresponds to the imposed electric displacement field $D_{z}=0.031 \mathrm{C}$ $\mathrm{m}^{-2}$ (comparable to the displacement field next to moderately charged colloids or lamellar liquid crystals ${ }^{56,57}$ ); $D_{z} \varepsilon_{\mathrm{o}}{ }^{-1}$ $\sim 0.35 \mathrm{~V} \AA^{-1}$. The actual, dielectrically screened field $E_{z}$ $=E_{z}^{0}(0,0)-P_{z} \varepsilon_{0}^{-1} \ll E_{z}^{0}(0,0)$. Here $E_{z}^{0}(0,0)=D_{z} \varepsilon_{o}^{-1}$ and $P_{\mathrm{Z}}$ is the medium polarization density along $z$ direction. Since $E_{z}$ is not known before the actual computation, ${ }^{58} D_{z}$ is used as input quantity. In this work we estimate the actual (dielectrically screened) field strength $E_{z}(z)$ from the orientational polarization of water in the direction of the field.
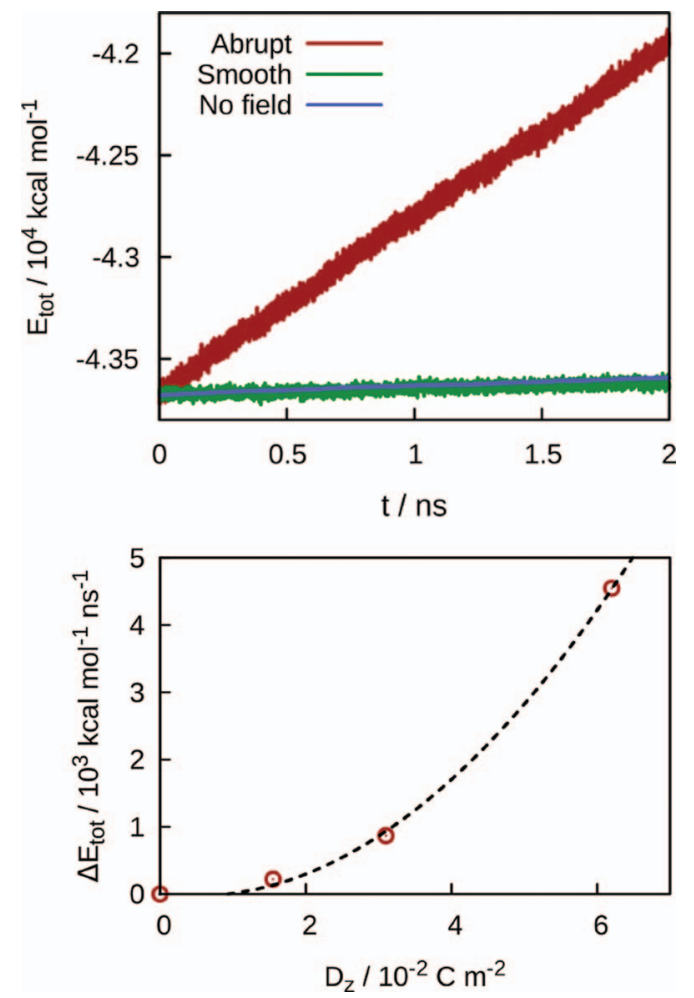

FIG. 3. (Top) Time evolution of the total energy of the simulated system comprised of a field-free or field-exposed confinement surrounded by $\sim 5$ $\times 10^{3}$ water molecules during $N V E$ simulation. Blue line: no field, green: electric displacement field $D_{\mathrm{z}}=0.031 \mathrm{C} \mathrm{m}^{-2}$ with smooth truncation, red: electric displacement field $D_{\mathrm{z}}=0.031 \mathrm{C} \mathrm{m}^{-2}$ with abrupt truncation. (Bottom) Energy drift under abruptly discontinued field as a function of the strength of the electric displacement field $D_{\mathrm{z}}$ from $N V E$ simulation (circles). Dashed line: quadratic fit of simulation results.

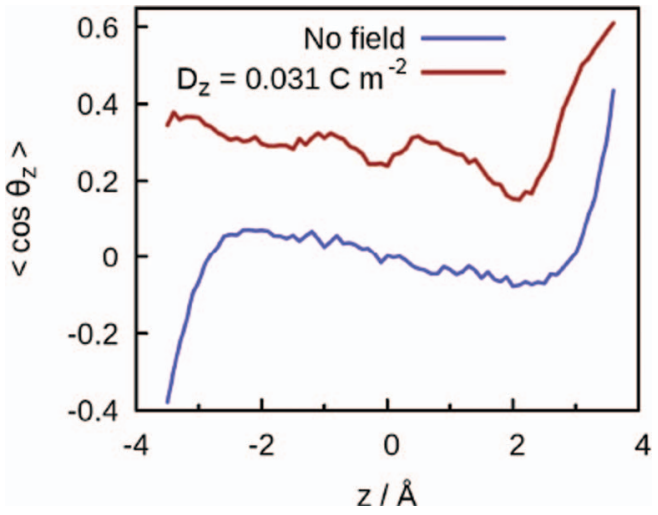

FIG. 4. Average cosine of the angle between water-molecule dipoles and the direction of the field in the confinement. Blue line: spontaneous polarization of water along $z$ direction (normal to confinement walls) in the absence of external field and red line: at electric displacement field $D_{\mathrm{z}}=0.031 \mathrm{C} \mathrm{m}^{-2}$.

Fig. 4 illustrates the profile of $\cos \theta_{z}=\left\langle\mu_{z}\right\rangle /|\mu|$ across the confinement in the absence (blue curve) or presence of external field. $\left\langle\mu_{z}\right\rangle$ is the average $z$ component of molecular dipoles in $z$ direction, normal to the confinement plates. Nonzero $\left\langle\mu_{z}\right\rangle$ in the field-free system reflects spontaneous polarization of interfacial water ${ }^{59-62}$ optimizing hydrogen bonding ${ }^{59,60}$ and dipole/wall image ${ }^{56,63}$ interaction. It has been established that $\cos \theta_{\mathrm{z}}$ in water follows the Langevin-Debye relation $\cos \theta_{\mathrm{z}}$ $\sim \mathrm{L}(|E \mu| / \mathrm{k} T)$ where $\mathrm{L}(x)=\operatorname{coth}(x)-x^{-1}$ and $\mathrm{k}$ is the Boltzmann constant. ${ }^{5,64,65}$ Fig. 5 shows $\cos \theta_{\mathrm{z}}$ as a function of total field $E_{z}$ from our simulations in bulk water with 3D Ewald sums and conducting boundary conditions. Using these data and the simulated profiles $\cos \theta_{\mathrm{z}}(z)$ in the confinement (Fig. 4), we estimate the effective field $E_{\mathrm{z}}(z)$ as the fieldincrement corresponding to the change in $\cos \theta_{\mathrm{z}}$ in Fig. 5 from the confinement value in the absence of the field, $\cos \theta_{\mathrm{z}}(z, 0)$, to its value under the imposed field $D_{\mathrm{z}}, \cos \theta_{\mathrm{z}}\left(z, D_{\mathrm{z}}\right)$ (Fig. 4). The resulting profile $E_{\mathrm{z}}(z)$ is illustrated in Fig. 6. The average field across the aqueous slab, $\bar{E}_{z} \leq 0.02 \mathrm{~V} \AA^{-1}$, is over an order of magnitude weaker than the unscreened input field $D_{z} \varepsilon_{o}{ }^{-1}$ suggesting the slab average of the inverse dielectric response function ${ }^{66}$ along $z$ axis, $\overline{\varepsilon_{z}^{-1}}$, of $\approx 15^{-1}(1 \pm 20 \%)$. The comparatively high value of $\overline{\varepsilon_{z}^{-1}}$, associated with the

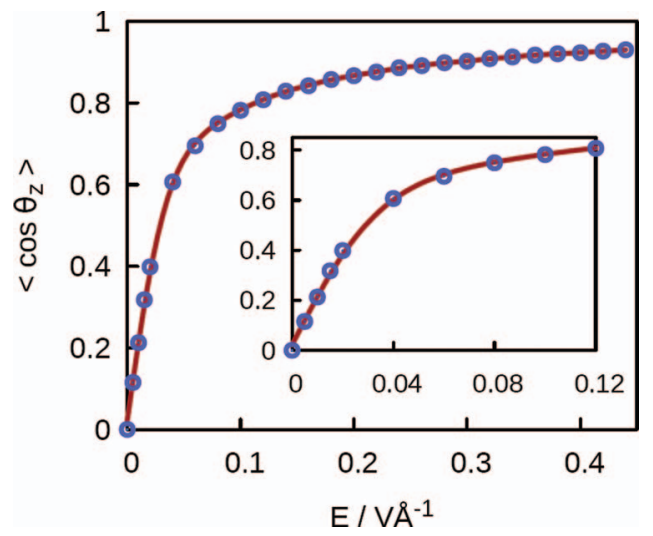

FIG. 5. Average cosine of the angle between molecular dipoles and the direction of the field in bulk water as a function of the actual field strength $E=|E|$. 


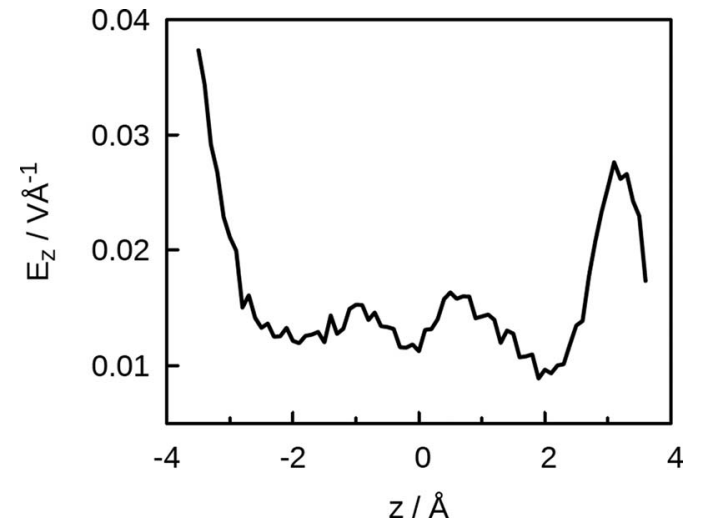

FIG. 6. Profile of the average strength of the dielectrically screened field $E_{\mathrm{z}}$ across a $12 \AA$ wide aqueous confinement at electric displacement field $D_{\mathrm{z}}=0.031 \mathrm{C} \mathrm{m}^{-2}\left(D_{z} \varepsilon_{\mathrm{o}}^{-1}=0.35 \mathrm{~V}^{-1}\right)$.

proximity of the low permittivity interfacial regions, agrees well with previous estimates for ion channels of comparable width. ${ }^{1}$ The electric-field profile $E_{\mathrm{z}}(z)$, Fig. 6 , is consistent with the oscillatory dielectric response profile, $\varepsilon_{z}^{-1}(z)$, determined in a recent study of interfacial permittivity at similar conditions. ${ }^{66}$

The time evolution of the total energies of both the fieldfree system (blue) and system $S$ (smoothly truncated field, green) in Fig. 3 (top) show identical, negligibly small drift of $\sim 0.06 \%$ per ns, which is apparently not related to system's electrostatics. The two curves essentially overlap because the average molecule/field interaction $\left\langle U_{E}\right\rangle \sim-\mu E_{\mathrm{z}}\left\langle\cos \theta_{\mathrm{z}}\right\rangle$ of $\sim-0.066 \mathrm{k} T$ is comparatively small and affects only about $7 \%$ of the molecules in the box. The curve describing system $A$ (red), on the other hand, shows a drift of $\sim 2 \%$ of total energy per ns. The rise in the energy reflects the energy increase of the order of $-\left\langle U_{E}\right\rangle$ for each escape of a (partially aligned) molecule from the field. The increase takes place because continuous molecular equations of motion cannot account for the impulse of force on the escaping molecules and concomitant kinetic energy reduction. For molecules entering the confinement, the effect is less significant as the environment molecules have near random orientations, i.e., $\left\langle\cos \theta_{z}\right\rangle$ close to zero except for a small fraction of molecules recrossing into the confinement before they had a chance to random-

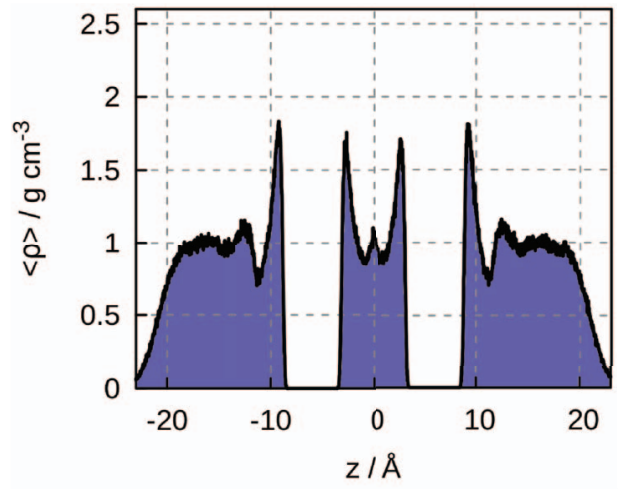

(a) NVE - No field ize their orientation; typical relaxation time for this process is between 5 and 8 ps. ${ }^{62,67-69}$ The growth of the energy drift with the field strength shown in Fig. 3 (bottom) conforms to this picture. For present fields, the alignment of water molecules grows essentially linearly with the field, $\left\langle\cos \theta_{\mathrm{z}}\right\rangle \propto\left|E_{\mathrm{z}}\right|^{13,64}$ (see also Fig. 5) and $\left\langle U_{E}\right\rangle \propto E_{z}^{2}$. According to Fig. 3 (bottom), the energy drift increases with the square of the applied field. This confirms that the drift is proportional to $\left\langle U_{E}\right\rangle$, and the proportionality factor is indicative of the rate of molecular escapes from the region under the field. The negligible energy drift in smoothly truncated fields (system $S$ ) conforms to the above interpretation.

\section{B. Water structure and barostat function}

In contrast to straightforward MD integration in the system with smooth electric field cutoff (system $S$ ), a rigorous integration in the presence of discontinuous field (system $A$ ) would require an inclusion of the impulse term for molecules crossing the discontinuity plane. By facilitating the molecules' escape from the field, the omission of this term can bias the partitioning of water between the bulk reservoir and the confinement, and can affect the performance of the barostat intended to maintain constant chemical potential of water in the reservoir and system as a whole. We evaluate the magnitude of both effects by comparing the results for water structure in systems with no field (Fig. 7) and those in which the confinement is spanned by the applied field, smoothly $(S)$ or abruptly $(A)$ truncated at the confinement border (Figs. 8 and 9). In each of the three systems, ambient (near zero) pressure was maintained by two different methods. We used pressure buffering method based on vapor/liquid coexistence ${ }^{26,27}$ in $N V E$ ensemble, or conventional Nose-Hoover barostat ${ }^{25}$ in $N P T$ simulations. Density profiles of water, $\rho_{\mathrm{H}_{2} \mathrm{O}}(z)$, for both types of imposed conditions are presented in Fig. 8 for $D_{z}$ $=0.031 \mathrm{C} \mathrm{m}^{-2}$ and in Fig. 9 for $D_{z}=0.062 \mathrm{C} \mathrm{m}^{-2}$. Average densities inside the confinement, and in the reservoir, are compared in Table I. Density profiles and associated density averages for field free systems as well as systems with smoothly truncated confinement field show no differences between the two ensemble types, each relying on a different method of barostating. Introduction of electric field inside the

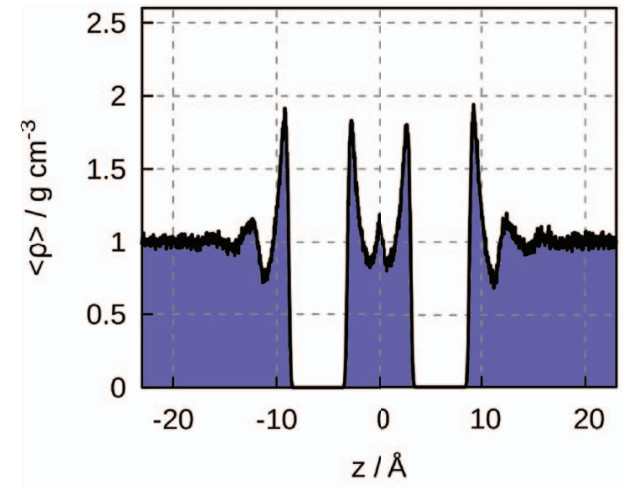

(b) NPT - No field

FIG. 7. Water density profiles in the central portion $\left(r \leq r_{\text {in }}\right)$ of the reservoir/confinement system in the absence of external field. (a) $N V E$ simulation with pressure-buffer barostating. See caption of Fig. 1. (b) NPT simulation using Nose-Hoover barostat/thermostat. 


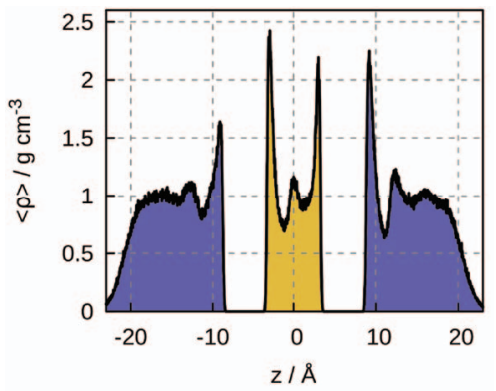

(a) NVE - Smooth

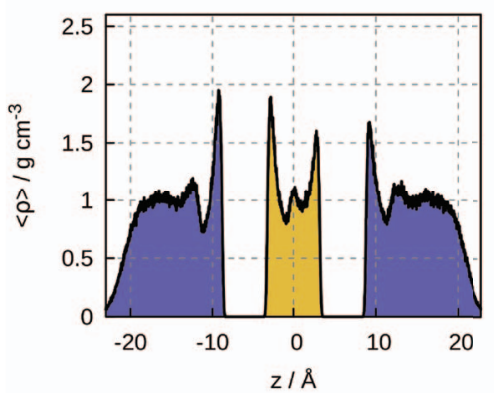

(c) NVE - Abrupt

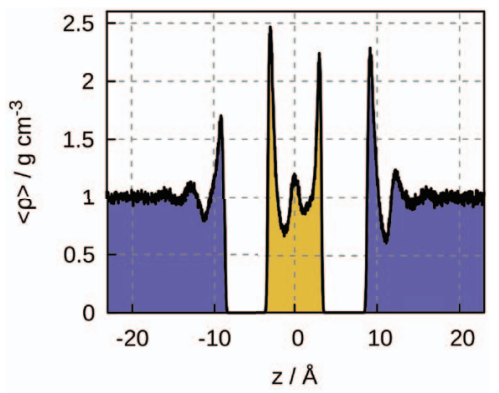

(b) NPT - Smooth

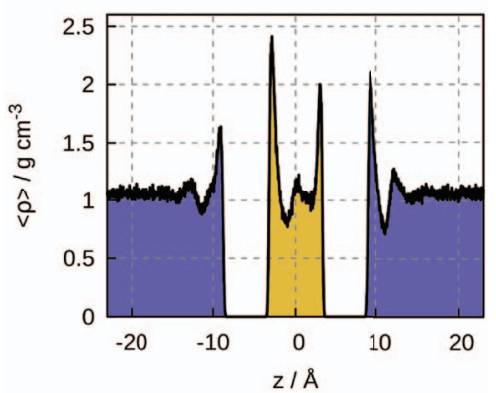

(d) NPT - Abrupt

FIG. 8. Water density profiles in the central portion of the reservoir/confinement system at electric displacement field across the confinement $D_{\mathrm{z}}=0.031 \mathrm{C}$ $\mathrm{m}^{-2}$ with smoothly (a) and (b) or abruptly truncated field (c) and (d). Left: $N V E$ simulation with pressure-buffer barostating. Right: $N P T$ simulation using Nose-Hoover barostat/thermostat. Consistent with Fig. 1, blue and yellow colors denote field-free and field-exposed regions, respectively.

confinement consistently increases local density. This behavior conforms to classical electrostriction demonstrated in previous $\mathrm{MD}^{1}$ and Monte Carlo studies. ${ }^{4,6,7,13}$ An opposite trend has been reported in an earlier MD study based on discontinuous field truncation with no force impulse correction. ${ }^{5}$ Us-

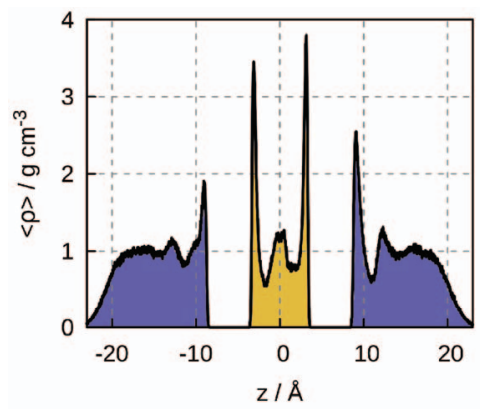

(a) NVE - Smooth ing the same approximation, our results for abruptly truncated field (system $A$ ) in Figs. 8 and 9 show a density reduction, which intensifies with increasing strength of the applied field. As already pointed out, we attribute the reduction to the neglect of the force impulse countering the molecules' attempts

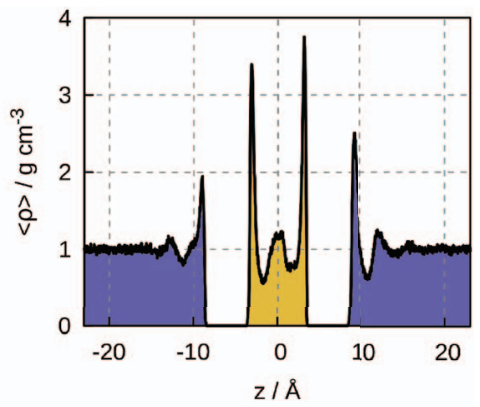

(b) NPT - Smooth

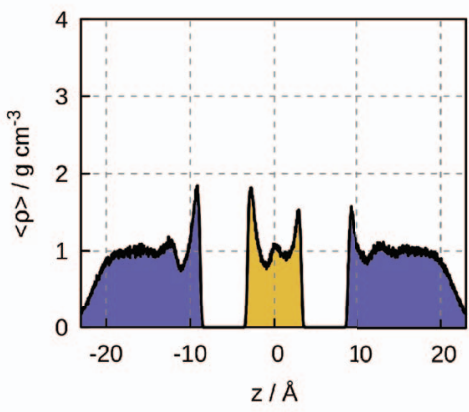

(c) NVE - Abrupt

FIG. 9. Water density profiles in the central portion of the reservoir/confinement system at electric displacement field $D_{\mathrm{z}}=0.062 \mathrm{C} \mathrm{m}^{-2}$ with smooth (a) and (b) or abrupt (c) truncation at the confinement border. Systems (a) and (c): NVE simulation with pressure-buffer barostating. System (b): NPT simulation using Nose-Hoover barostat/thermostat. Blue and yellow colors denote field-free and field-exposed regions. 
TABLE I. Average densities of water in the field-exposed confinement, $\rho_{\mathrm{H}_{2} \mathrm{O}}^{c}$, (relative to the field-free confinement), and in the field-free bath at ambient conditions, $\rho_{\mathrm{H}_{2} \mathrm{O}}^{b}$, from NVE simulations with pressure buffering, or NPT simulations with Nose-Hoover barostat/thermostat. Results for smoothly (system $S$ ) and abruptly (system $A$ ) truncated fields across the confinement are given for field strengths corresponding to electric displacement fields $D_{z}=0.031$ and $0.062 \mathrm{C} \mathrm{m}^{-2}$.

\begin{tabular}{|c|c|c|c|c|c|}
\hline \multicolumn{2}{|c|}{ System type } & \multicolumn{2}{|c|}{$N V E$} & \multicolumn{2}{|c|}{$N P T$} \\
\hline$D_{z}\left(\mathrm{C} \mathrm{m}^{-2}\right)$ & Field truncation & $\frac{\rho_{H_{2} O^{(}}^{c}\left(D_{z}\right)}{\rho_{H_{2} O^{2}}^{c}(0)}$ & $\rho_{\mathrm{H}_{2} \mathrm{O}}^{b}\left(\mathrm{~g} \mathrm{~cm}^{-3}\right)$ & $\frac{\rho_{H_{2} O}^{c}{ }^{\left(D_{z}\right)}}{\rho_{H_{2}}^{c} O^{(0)}}$ & $\rho_{\mathrm{H}_{2} \mathrm{O}}^{b}\left(\mathrm{~g} \mathrm{~cm}^{-3}\right)$ \\
\hline 0 & $\ldots$ & 1.00 & $0.98 \pm 0.03$ & 1.00 & $0.99 \pm 0.03$ \\
\hline 0.031 & Smooth $(S)$ & $1.03 \pm 0.01$ & $0.99 \pm 0.03$ & $1.04 \pm 0.02$ & $0.99 \pm 0.03$ \\
\hline 0.062 & Smooth $(S)$ & $1.08 \pm 0.02$ & $0.99 \pm 0.03$ & $1.09 \pm 0.02$ & $0.98 \pm 0.03$ \\
\hline 0.031 & Abrupt $(A)$ & $0.95 \pm 0.02$ & $0.99 \pm 0.03$ & $0.99 \pm 0.02$ & $1.06 \pm 0.03$ \\
\hline 0.062 & Abrupt $(A)$ & $0.90 \pm 0.02$ & $0.97 \pm 0.03$ & $0.92 \pm 0.07$ & $1.12 \pm 0.03$ \\
\hline
\end{tabular}

to escape from the field-exposed region. In view of the complications associated with the incorporation of the rigorous discontinuous MD algorithm ${ }^{54}$ in a system where long-ranged forces are present simultaneously, the smooth truncation of the electric field represents the preferable alternative.

Our comparison between two barostating methods also unveils a considerable barostat dependence of water structure in the presence of the abruptly truncated field. The differences, attributed to the omission of appropriate discontinuity pressure-correction ${ }^{25}$ in system $A$ include a density reduction inside the confinement and increase outside of it. The differences increase with the strength of the field. In the strongest field we consider, $D_{\mathrm{z}}=0.062 \mathrm{C} \mathrm{m}^{-2}$, system $A$ displays the onset of liquid/vapor separation in the confined phase. In this case, large density fluctuations are present in both, the confined and bulk regions, and we could not obtain statistically meaningful density profiles for this system.

\section{System variations}

In all the above examples, we used the decay function described by Eq. (5). To examine possible influence of specific form of the field decay, a part of our calculations was repeated using a different form of the decay function $f(r)$ inside the interval $r_{\text {in }} \leq r \leq r_{\text {out }}$,

$$
f\left(r_{s}\right)=\exp \left(-\frac{r_{s}^{2}}{1-r_{s}^{2}}\right), \quad r_{s}=\frac{r-r_{\text {in }}}{r_{\text {out }}-r_{\text {in }}} .
$$

Despite a considerable difference between decay functions in Eqs. (5) and (10), neither this change nor moderate variations in the length of the decay interval $\left(r_{\text {out }}-r_{\text {in }}\right)$ produced noteworthy differences in obtained structures outside the interval itself. Similarly, energy conservation remains excellent regardless of the above modification in the form of smooth truncation. Insensitivity on the form of $f(r)$ has been recently confirmed in calculations where neat water was replaced by 1-2 $\mathrm{M} \mathrm{NaCl}$ solution. ${ }^{70}$

Density profiles in Figs. 7 and 8, as well as the related results from Ref. 5, indicate the presence of water-water correlations across the confinement plates. To examine the importance of these correlations on the behavior of the confined phase, we repeated the calculations described in Fig. 7 using essentially identical system parameters, but for markedly thicker confinement walls. These walls were com- prised of model butylated graphane, whose wetting properties we described in previous works. ${ }^{50,51}$ The separation between opposite hydration layers across a graphane plate was close to $1.5 \mathrm{~nm}$, which is more than double the distance in the case of graphene. Nonetheless, all qualitative features of graphene system and its response to the applied field, including the distinction between smooth and abrupt field truncations remained unchanged, confirming the observed behavior to be robust with respect to the details of model system.

\section{CONCLUDING REMARKS}

We use MD simulations to examine effects of electric field in aqueous confinements open to an unperturbed environment at ambient conditions. In the confinement interior, the direction of the applied field is normal to confinement walls. The decay of the applied field at the confinement border, however, implies the existence of a nonvanishing component of local field in lateral direction. Depending on the orientation of water molecule at the confinement border, this field can attract a dipolar molecule into or repel it away from the field-exposed region. Because of biased orientations of confined molecules, the net effect is attractive. The effect can be readily captured in MD simulation in the case of smooth field truncation but would require the incorporation of the discontinuous MD algorithm ${ }^{54}$ in systems with abrupt field decay. The omission of the lateral impulse term, required in rigorous MD integration, manifests in the energy drift of an isolated system and leads to an underestimate of liquid density in the field-exposed region. Calculated pressure and Nose-Hoover barostat can also be affected. With proper treatment of the field-decay region at the confinement border, the imposition of external field across the confinement in equilibrium with a field-free aqueous reservoir consistently results in density increase in analogy with electrostriction in bulk water. A contradictory observation ${ }^{5}$ in the same type of system may be explained by the neglect of the lateral field effect discussed above.

The field-induced density ${ }^{12,15}$ and critical point ${ }^{12}$ depression observed in MD simulations, both in the bulk and confined aqueous phases, on the other hand, pertain to different imposed conditions. The key differences include the variation of the chemical potential with the field strength, the absence of a field-free reservoir supplying additional 
molecules, and the extension of uniform electric displacement field across the entire system under consideration. The critical role of imposed conditions on the magnitude and sign of the field-induced density changes has been highlighted in recent works. ${ }^{13,14}$

\section{ACKNOWLEDGMENTS}

While carrying out this work, D.V. and A.L. were supported by the National Science Foundation (CHE-1213814) and D.B. was supported by the U.S. Department of Energy, Basic Energy Sciences (DE-SC 0004406). We also acknowledge computer time allocations from the National Energy Research Scientific Computing Center (NERSC), which is supported by the Office of Science of the U.S. Department of Energy (DEAC02-05CH11231), and the Extreme Science and Engineering Discovery Environment XSEDE), which is supported by NSF Grant No. OCI-1053575.

${ }^{1}$ J. Dzubiella and J. P. Hansen, J. Chem. Phys. 122, 234706 (2005).

${ }^{2}$ S. N. Smirnov, I. V. Vlassiouk, and N. V. Lavrik, ACS Nano 5, 7453 (2011).

${ }^{3}$ J. Dzubiella and J. P. Hansen, J. Chem. Phys. 121, 5514 (2004).

${ }^{4}$ S. Vaitheeswaran, J. C. Rasaiah, and G. Hummer, J. Chem. Phys. 121, 7955 (2004).

${ }^{5}$ S. Vaitheeswaran, H. Yin, and J. C. Rasaiah, J. Phys. Chem. B 109, 6629 (2005).

${ }^{6}$ D. Bratko, C. D. Daub, K. Leung, and A. Luzar, J. Am. Chem. Soc. 129, 2504 (2007).

${ }^{7}$ D. Bratko, C. D. Daub, and A. Luzar, Faraday Discuss. 141, 55 (2009).

${ }^{8}$ M. von Domaros, D. Bratko, B. Kirchner, and A. Luzar, J. Phys. Chem. C 117, 4561 (2013).

${ }^{9}$ R. K. Kalluri, M. M. Biener, M. E. Suss, M. D. Merrill, M. Stadermann, J. G. Santiago, T. F. Baumann, J. Biener, and A. Striolo, Phys. Chem. Chem. Phys. 15, 2309 (2013).

${ }^{10}$ S. Gekle and R. R. Netz, J. Chem. Phys. 137, 104704 (2012).

${ }^{11}$ R. Zangi and A. E. Mark, J. Chem. Phys. 120, 7123 (2004).

${ }^{12}$ R. Srivastava, J. K. Singh, and P. T. Cummings, J. Phys. Chem. C 116, 17594 (2012).

${ }^{13}$ D. Bratko, C. D. Daub, and A. Luzar, Phys. Chem. Chem. Phys. 10, 6807 (2008).

${ }^{14}$ C. D. Daub, D. Bratko, and A. Luzar, Top. Curr. Chem. 307, 155 (2012).

${ }^{15}$ J. L. England, S. Park, and V. S. Pande, J. Chem. Phys. 128, 044503 (2008).

${ }^{16}$ M. R. Powell, L. Cleary, M. Davenport, K. J. Shea, and Z. S. Siwy, Nat. Nanotechnol. 6, 798 (2011).

${ }^{17}$ B. X. Xu, Y. Qiao, Q. L. Zhou, and X. Chen, Langmuir 27, 6349 (2011).

${ }^{18}$ L. Figueras and J. Faraudo, Mol. Simul. 38, 23 (2012).

${ }^{19}$ J. Dzubiella, R. J. Allen, and J. P. Hansen, J. Chem. Phys. 120, 5001 (2004).

${ }^{20}$ T. A. Ho, D. Argyris, D. R. Cole, and A. Striolo, Langmuir 28, 1256 (2012).

${ }^{21}$ R. K. Kalluri, D. Konatham, and A. Striolo, J. Phys. Chem. C 115, 13786 (2011).

22 J. I. Siepmann and M. Sprik, J. Chem. Phys. 102, 511 (1995).

${ }^{23}$ S. K. Reed, O. J. Lanning, and P. A. Madden, J. Chem. Phys. 126, 084704 (2007).

${ }^{24}$ C. Merlet, M. Salanne, B. Rotenberg, and P. A. Madden, J. Phys. Chem. C 115, 16613 (2011)

${ }^{25}$ D. Frenkel and B. Smit, Understanding Molecular Simulation, from Algorithms to Applications (Academic, San Diego, 2002).

${ }^{26}$ P. G. Bolhuis and D. Chandler, J. Chem. Phys. 113, 8154 (2000).

${ }^{27}$ Y. J. Lei and Y. S. Leng, Langmuir 28, 3152 (2012).

${ }^{28}$ T. Werder, J. H. Walther, R. L. Jaffe, T. Halicioglu, and P. Koumoutsakos, J. Phys. Chem. B 107, 1345 (2003).
${ }^{29}$ N. Choudhury and B. M. Pettitt, J. Am. Chem. Soc. 127, 3556 (2005).

${ }^{30}$ H. Zhou, P. Ganesh, V. Presser, M. C. F. Wander, P. Fenter, P. R. C. Kent, D. E. Jiang, A. A. Chialvo, J. McDonough, K. L. Shuford, and Y. Gogotsi, Phys. Rev. B 85, 035406 (2012).

${ }^{31}$ C. Eun and M. L. Berkowitz, J. Phys. Chem. B 114, 13410 (2010).

${ }^{32}$ H. J. C. Berendsen, J. R. Grigera, and T. P. Straatsma, J. Phys. Chem. 91, 6269 (1987).

${ }^{33}$ B. Guillot, J. Mol. Liq. 101, 219 (2002).

${ }^{34}$ C. Vega, J. L. F. Abascal, M. M. Conde, and J. L. Aragones, Faraday Discuss. 141, 251 (2009).

${ }^{35}$ I. Bako, M. A. Ricci, P. G. Debenedetti, M. Rovere, M. Havenith, F. X. Coudert, A. Luzar, J. Klein, P. Jungwirth, T. Yamaguchi, M. Lee, J. Yarwood, A. Michaelides, M. Salmeron, P. Feibelman, C. Vega, A. Patel, A. Hodgson, J. Finney, P. Madden, K. Uosaki, J. Chen, W. Jhe, S. S. Perkin, B. Halle, S. J. Kim, C. Bain, H. Christenson, M. L. Rao, G. Held, L. Lyashchenko, R. von Klitzing, J. Van Der Niet, F. Hooton, M. Marques, D. Y. Wu, and S. Johnson, Faraday Discuss. 141, 443 (2009).

${ }^{36}$ O. Gereben and L. Pusztai, Chem. Phys. Lett. 507, 80 (2011).

${ }^{37}$ D. M. Huang, P. L. Geissler, and D. Chandler, J. Phys. Chem. B 105, 6704 (2001).

${ }^{38}$ M. Agarwal, M. P. Alam, and C. Chakravarty, J. Phys. Chem. B 115, 6935 (2011).

${ }^{39}$ J. L. Aragones, L. G. MacDowell, J. I. Siepmann, and C. Vega, Phys. Rev. Lett. 107, 155702 (2011).

${ }^{40}$ A. Luzar, D. Bratko, and L. Blum, J. Chem. Phys. 86, 2955 (1987).

${ }^{41}$ K. Lum and A. Luzar, Phys. Rev. E 56, R6283 (1997).

${ }^{42}$ K. Lum, D. Chandler, and J. D. Weeks, J. Phys. Chem. B 103, 4570 (1999).

${ }^{43}$ D. Bratko, R. A. Curtis, H. W. Blanch, and J. M. Prausnitz, J. Chem. Phys. 115, 3873 (2001).

${ }^{44}$ K. Leung, A. Luzar, and D. Bratko, Phys. Rev. Lett. 90, 065502 (2003).

${ }^{45}$ J. C. Rasaiah, S. Garde, and G. Hummer, Annu. Rev. Phys. Chem. 59, 713 (2008).

${ }^{46}$ S. Sharma and P. G. Debenedetti, J. Phys. Chem. B 116, 13282 (2012).

${ }^{47}$ A. Luzar and K. Leung, J. Chem. Phys. 113, 5836 (2000).

${ }^{48}$ A. Luzar, J. Phys. Chem. B 108, 19859 (2004).

${ }^{49}$ B. J. Berne, J. D. Weeks, and R. H. Zhou, Annu. Rev. Phys. Chem. 60, 85 (2009).

${ }^{50}$ D. Vanzo, D. Bratko, and A. Luzar, J. Chem. Phys. 137, 034707 (2012).

${ }^{51}$ D. Vanzo, D. Bratko, and A. Luzar, J. Phys. Chem. C 116, 15467 (2012).

${ }^{52}$ S. Plimpton, J. Comput. Phys. 117, 1 (1995).

${ }^{53}$ I. C. Yeh and M. L. Berkowitz, J. Chem. Phys. 111, 3155 (1999).

${ }^{54}$ B. J. Alder and T. E. Wainwright, J. Chem. Phys. 31, 459 (1959).

${ }^{55}$ A. V. Smith and C. K. Hall, J. Mol. Biol. 312, 187 (2001).

${ }^{56}$ D. Bratko, B. Jonsson, and H. Wennerstrom, Chem. Phys. Lett. 128, 449 (1986).

${ }^{57}$ J. Z. Wu, D. Bratko, H. W. Blanch, and J. M. Prausnitz, J. Chem. Phys. 111, 7084 (1999).

${ }^{58}$ I. C. Yeh and M. L. Berkowitz, J. Chem. Phys. 110, 7935 (1999).

${ }^{59}$ A. Luzar, S. Svetina, and B. Zeks, J. Chem. Phys. 82, 5146 (1985).

${ }^{60}$ C. Y. Lee, J. A. McCammon, and P. J. Rossky, J. Chem. Phys. 80, 4448 (1984).

${ }^{61}$ J. C. Shelley and G. N. Patey, Mol. Phys. 88, 385 (1996).

${ }^{62}$ G. Stirnemann, P. J. Rossky, J. T. Hynes, and D. Laage, Faraday Discuss. 146, 263 (2010)

${ }^{63}$ S. J. Miklavcic, Phys. Rev. E 56, 1142 (1997).

${ }^{64}$ G. Sutmann, J. Electroanal. Chem. 450, 289 (1998).

${ }^{65}$ J. Mittal and G. Hummer, Faraday Discuss. 146, $341(2010)$.

${ }^{66}$ D. J. Bonthuis, S. Gekle, and R. R. Netz, Langmuir 28, 7679 (2012).

${ }^{67}$ I. M. Svishchev, P. G. Kusalik, J. Wang, and R. J. Boyd, J. Chem. Phys. 105, 4742 (1996).

${ }^{68}$ D. van der Spoel, P. J. van Maaren, and H. J. C. Berendsen, J. Chem. Phys. 108, 10220 (1998).

${ }^{69}$ A. Y. Zasetsky, S. V. Petelina, A. K. Lyashchenko, and A. S. Lileev, J. Chem. Phys. 133, 134502 (2010).

${ }^{70}$ D. Vanzo, A. Luzar, and D. Bratko, "Reversibility of electric-field induced phase transitions in nanoconfined water and $\mathrm{NaCl}$ solutions" (unpublished). 